\title{
Stabilization of Continuous-Time Random Switching Systems via a Fault-Tolerant Controller
}

\author{
Guoliang Wang and Zhiqiang Li \\ School of Information and Control Engineering, Liaoning Shihua University, Fushun, Liaoning 113001, China \\ Correspondence should be addressed to Guoliang Wang; gliangwang@aliyun.com
}

Received 18 February 2017; Accepted 13 March 2017; Published 20 March 2017

Academic Editor: Mohammad D. Aliyu

Copyright (C) 2017 Guoliang Wang and Zhiqiang Li. This is an open access article distributed under the Creative Commons Attribution License, which permits unrestricted use, distribution, and reproduction in any medium, provided the original work is properly cited.

\begin{abstract}
This paper focuses on the stabilization problem of continuous-time random switching systems via exploiting a fault-tolerant controller, where the dwell time of each subsystem consists of a fixed part and random part. It is known from the traditional design methods that the computational complexity of LMIs related to the quantity of fault combination is very large; particularly system dimension or amount of subsystems is large. In order to reduce the number of the used fault combinations, new sufficient LMI conditions for designing such a controller are established by a robust approach, which are fault-free and could be solved directly. Moreover, the fault-tolerant stabilization realized by a mode-independent controller is considered and suitably applied to a practical case without mode information. Finally, a numerical example is used to demonstrate the effectiveness and superiority of the proposed methods.
\end{abstract}

\section{Introduction}

As we know, Markovian jump system (MJS) is a particular kind of hybrid systems, which contains two parts of mechanism. One mechanism is related to system state over time, and another mechanism is named to operation mode and derived from the finite set of discrete time Markov jump parameters. Because of experiencing random characteristics, Markovian jump system is greatly suitable to describe many practical systems whose parameters or structures change abruptly, such as [1-3]. In the past few years, a lot of topics on many kinds of MJSs have been widely studied, such as stability analysis [4-7], stabilization [8-11], $H_{\infty}$ control and filtering [12-14], output control [15-17], adaptive control [18$20]$, synchronization [21, 22], and robust control [23-26].

On the other hand, any system is inevitable to have faults in practice. It is necessary and meaningful to study the related problems of system experiencing faults. Fault-tolerant control problem [27-29] is when the faults of actuator, sensor, or internal component occur, the closed-system is still stable and has ideal characteristics. It is known that the classical fault-tolerant control methods may be divided into two categories: passive and active fault-tolerant control methods. Passive fault-tolerant control method is to use a fixed controller which ensures the closed-loop system insensitive to some specific faults. In other words, it could maintain the system stable. Therefore, this strategy is similar to the robust control technique [30,31]. On the contrary, active fault-tolerant control method needs to reconstruct the controller design and reschedule the control law. In other words, according to the characteristics of expectation, we need to design a new control system after the faults occurred $[32,33]$. Compared with the active fault-tolerant control method, the passive fault-tolerant control method does not need the real-time fault information or adjust the structure of controller online. In this sense, it is said that passive faulttolerant control method is relatively simple to implement. When the underlying system is referred to Markovian jump system, some results about fault-tolerant control were given in [34-36]. By investigating such references, it is seen that the considered problems and studied methods between such cited references and this paper are quite different. Firstly, the considered system is different from the traditional Markovian jump system, where the dwell time considered here consists of a fixed part and random part. Secondly, the fault considered here is described by using a binary structured uncertainty 
rather than that simply described by a vector in some existing references. In fact, this structure is more clear to describe the fault. Compared with the traditional methods dealing with the fault, the method to be presented has a better relaxation and is more applicable. Thirdly, but not the last, because of the fault of controller described by a binary structured uncertainty, the quantity of fault combination will be very large and have large computation complexity. Moreover, due to the fixed and random dwell time contained simultaneously, how to make the existence conditions for such a fault-tolerant controller within LMI framework and have a concise form are also necessarily studied. It is said that the abovementioned problems not only are important in theory, but also have practical applications. For example, from [37], it was shown that the helicopter system could be modeled as an Markovian jump system, whose dynamic characteristics are clearly described by a Markov chain with three different states according to airspeeds of 135 (nominal value), 60, and 170 knots. Moreover, the helicopter system is also inevitable to have kinds of faults due to the internal components faults happening or the external environment changing in practice. In order to guarantee it still works when these faults occur, a better and necessary scheme is to design an effective faulttolerant controller. On the other hand, though the switching of helicopter among such three modes satisfies a Markov process, it is more reasonable that each subsystem is likely to hold for a period of time. In other words, there will be a fixed and random dwell time in each subsystem. Finally, but not the last, it is also important that the desired control method should be with less computation complexity and easily realized. Based on these explanations, it is said that it is significative to design a fault-tolerant controller for Markovian jump systems experiencing forced dwell time and also has practical significance. To the best of our knowledge, very few results are available to design fault-tolerant controller for random switching system. All the facts motivate the current research.

In this paper, the stabilization problem of continuoustime random switching systems closed by a fault-tolerant controller will be studied, whose conditions are presented in terms of LMIs and without any fault. The main contributions of this paper are summarized as follows: (1) A kind of faulttolerant controller is proposed to stabilize a continuous-time random switching system which contains fixed and random dwell time simultaneously, whose conditions are obtained by exploiting a robust method; (2) the sufficient conditions for the desired controller are presented with LMI forms and fault-free, which could be solved directly; (3) due to the results without any fault, the complexity of computation will be smaller than ones obtained by the traditional methods; (4) because of the given conditions being LMIs, the existence conditions for fault-tolerant controller without any mode operation are obtained easily.

Notation. $\mathbb{R}^{n}$ denotes the $n$-dimensional Euclidean space; $\mathbb{R}^{q \times n}$ is the set of all $q \times n$ real matrices. $\|\cdot\|$ refers to the Euclidean vector norm or spectral matrix norm. $\Omega$ is the sample space, $\mathscr{F}$ is the $\sigma$-algebras of subsets of the sample space, and $\mathbb{P}$ is the probability measure on $\mathscr{F}$. In symmetric block matrices, we use “*” as an ellipsis for the terms induced by symmetry, $\operatorname{diag}\{\cdots\}$ for a block-diagonal matrix, and $(M)^{\star} \triangleq$ $M+M^{T}$.

\section{Problem Formulation}

Consider a class of switched linear systems defined on a complete probability space $(\Omega, \mathscr{F}, \mathbb{P})$ and described as

$$
\dot{x}(t)=A_{\eta(t)} x(t)+B_{\eta(t)} u(t),
$$

where $x(t) \in \mathbb{R}^{n}$ is the system state vector, $u(t) \in \mathbb{R}^{q}$ indicates the control input vector, and $\eta(t) \in \mathbb{S} \triangleq\{1,2, \ldots, N\}$ represents the switching signal and determines the current system operation mode. For any $\eta(t)=i \in \mathbb{S}, A_{\eta(t)}=A_{i}$ and $B_{\eta(t)}=B_{i}$ are known matrices of compatible dimensions. Time instant $t_{k}$ represents the current operation mode of the system to another operation mode. The parameter $d_{i}>0$ represents a fixed dwell time of system (1) with mode $i$. If the system occurs to interval $\left[t_{k}, t_{k}+d_{i}\right)$, where there is no switching, it will surely follow

$$
\operatorname{Pr}(\eta(t+h)=j \mid \eta(t)=i)= \begin{cases}0, & \text { if } j \neq i \\ 1, & \text { if } j=i\end{cases}
$$

where $h$ represents a very short amount of time and satisfies $\lim _{\Delta t \rightarrow 0^{+}}(o(h) / h)=0$. For the time interval $\left[t_{k}, t_{k}+d_{i}\right)$, if $t \geq t_{k}+d_{i}$, at this time the mode switching follows the mode transition probabilities with TRM $\Pi \triangleq\left(\pi_{i j}\right) \in \mathbb{R}^{N \times N}$ given by

$$
\begin{aligned}
\operatorname{Pr} & (\eta(t+h)=j \mid \eta(t)=i) \\
& = \begin{cases}\pi_{i j} h+o(h), & i \neq j \\
1+\pi_{i i} h+o(h), & i=j,\end{cases}
\end{aligned}
$$

where $h>0, \pi_{i j} \geq 0$, if $i \neq j$, and $\pi_{i i}=-\sum_{j \neq i} \pi_{i j}$.

In this paper, the designed state feedback controller may have faults, which is referred to be a fault-tolerant controller (FTC) and described by

$$
u(t)=\Delta(t) K_{\eta(t)} x(t)
$$

where $K_{\eta(t)}$ is the control gain to be determined. The parameter $\Delta(t)$ is a diagonal matrix and used to describe the controller fault happening or not. Its form is defined as

$$
\Delta(t) \in \Lambda=\left\{\Delta=\operatorname{diag}\left(\delta_{1}, \ldots, \delta_{q}\right) \mid \delta_{i} \in\{0,1\}\right\} .
$$

Particularly, we could clearly find that $\Delta(t)=I_{q}$ if there are no faults. It is seen that there are $2^{q}$ possible combinations representing the controller faults. Equivalently, $\Lambda$ has $2^{q}$ elements. Then, the resulting closed-loop system is rewritten to be

$$
\dot{x}(t)=\bar{A}_{\eta(t)} x(t),
$$

where $\bar{A}_{\eta(t)}=A_{\eta(t)}+B_{\eta(t)} \Delta(t) K_{\eta(t)}$. 
Remark 1. It is worth mentioning that the fault of controller (4) is described by a binary structured uncertainty. Compared with some existing references [27, 31, 32, 34-36] where the fault is modeled to be a vector, this formulation has a better description and more application scope. However, it is also seen that $2^{q}$ possible combinations are included to represent the controller faults. This will make the computation complexity very large; in particular the underlying system is a switching system with $N$ operation modes. Thus, how to reduce the complexity and make the obtained results with concise and easily solvable forms are necessary and meaningful problems.

Lemma 2 (see [4]). System (1) without any control is stochastically stable if and only if there exists matrix $P_{i}>0, i \in \mathbb{S}$, such that

$$
A_{i}^{T} P_{i}+P_{i} A_{i}+\pi_{i i} P_{i}+\sum_{j \in \mathbb{S}, j \neq i} \pi_{i j} e^{A_{j}^{T} d_{j}} P_{j} e^{A_{j} d_{j}}<0 .
$$

Lemma 3 (see [33]). Let $T_{1}=T_{1}^{T}, T_{2}, T_{3}$, and $T_{4}$ be given matrices with appropriate dimensions, where $\underline{\Delta}=\operatorname{diag}\left(\underline{\Delta}_{1}, \ldots\right.$, $\left.\underline{\Delta}_{m}\right)$ and $\bar{\Delta}=\operatorname{diag}\left(\bar{\Delta}, \ldots, \bar{\Delta}_{m}\right)$. Define $T_{4}^{\min }=I-T_{4} \underline{\Delta}, T_{4}^{\max }=$ $I-T_{4} \bar{\Delta}$, where $\operatorname{det}\left(T_{4}^{\min }\right) \neq 0$ and $\operatorname{det}\left(T_{4}^{\max }\right) \neq 0$ should be satisfied. Consider the following inequality

$$
\mathbb{T}(\Delta)=T_{1}+\left\{T_{2} \Delta\left(I-T_{4} \Delta\right)^{-1} T_{3}\right\}^{\star}<0
$$

holds for any $\Delta \in \Lambda$, if there exist matrices $S$ and $W$ such that

$$
\begin{aligned}
& {\left[\begin{array}{c|c}
T_{1}-T_{2}\left(\underline{\Delta} S \bar{\Delta}^{T}\right)^{\star} T_{2}^{T} & * \\
\hline T_{3}+\left(\underline{T}_{4} S \bar{\Delta}^{T}+\bar{T}_{4} S^{T} \underline{\Delta}^{T}-W\right) T_{2}^{T} & -\left(\underline{T}_{4} S \bar{T}_{4}^{T}+W T_{4}^{T}\right)^{\star}
\end{array}\right]} \\
& <0,
\end{aligned}
$$

where $S \in \mathbb{R}^{q \times q}$ and $W \in \mathbb{R}^{q \times q}$ satisfied $\Delta W+W^{T} \Delta^{T}=0$.

\section{Main Results}

Theorem 4. For given system (1), there exists an FTC (4) such that the resulting close-loop system (6) is stochastically stable, if for given scalars $\gamma_{i}>1$ and $\mu_{i}>0$, there exist matrices $X_{i}>0$, $Y_{i}$, and $S_{i}$, such that

$$
\begin{aligned}
& {\left[\begin{array}{cc}
\Omega_{i 1} & \Omega_{i 2} \\
* & \left(-S_{i}\right)^{\star}
\end{array}\right] \leq 0, } \\
& {\left[\begin{array}{cc}
\Psi_{i 1} & \Psi_{i 2} \\
* & \left(-S_{i}\right)^{\star}
\end{array}\right] \leq 0, } \\
{\left[\begin{array}{ccc}
\Phi_{i 1} & \Phi_{i 2} & \Omega_{i 2} \\
* & \Phi_{i 3} & 0 \\
* & * & -\left(S_{i}\right)^{\star}
\end{array}\right]<0, } & < \\
&
\end{aligned}
$$

where
Thus, the gain of controller (4) is computed as

$$
K_{i}=Y_{i} X_{i}^{-1}
$$

Proof. Replacing $A_{i}$ with $\bar{A}_{i}=A_{i}+B_{i} \Delta(t) K_{i}$ in (7), it is clearly known from Lemma 2 that the resulting closed-loop system is stochastically stable if the following condition is

$$
\left(\bar{A}_{i}^{T} P_{i}\right)^{\star}+\pi_{i i} P_{i}+\sum_{j \in \mathbb{S}, j \neq i} \pi_{i j} e^{\bar{A}_{j}^{T} d_{j}} P_{j} e^{\bar{A}_{j} d_{j}}<0, \quad \forall i \in \mathbb{S} .
$$

It is further guaranteed by

$$
\left(\bar{A}_{i}^{T} P_{i}\right)^{\star}+\pi_{i i} P_{i}+\sum_{j \in \mathbb{S}, j \neq i} \pi_{i j}\left\|e^{\bar{A}_{j}^{T} d_{j}}\right\|\left\|P_{j}\right\|\left\|e^{\bar{A}_{j} d_{j}}\right\| I<0 .
$$

For any matrix $A \in \mathbb{R}^{n \times n}, \forall t \geq 0$, it is claimed that $\left\|e^{A t}\right\| \leq$ $e^{\|A\| t}$. Then, it is obtained that

$$
\left(\bar{A}_{i}^{T} P_{i}\right)^{\star}+\pi_{i i} P_{i}+\sum_{j \in \mathbb{S}, j \neq i} \pi_{i j} e^{\left\|\bar{A}_{j}^{T}\right\| d_{j}}\left\|P_{j}\right\| e^{\left\|\bar{A}_{j}\right\| d_{j}} I<0 .
$$


By pre- and postmultiplying (18) with $X_{i}=P_{i}^{-1}$, it is get that

$$
\begin{aligned}
& \left(\bar{A}_{i} X_{i}\right)^{\star}+\pi_{i i} X_{i}+\sum_{j \in \mathbb{S}, j \neq i} \pi_{i j} X_{i} e^{\left\|\bar{A}_{j}^{T}\right\| d_{j}}\left\|X_{j}^{-1}\right\| e^{\left\|\bar{A}_{j}\right\| d_{j}} X_{i} \\
& \quad<0 .
\end{aligned}
$$

Based on Lemma 3, it is known that conditions (10), (11), and (12) imply the following conditions, respectively. That is,

$$
\Omega_{i 1}+\left(B_{i} \Delta Y_{i}\right)^{\star} \leq 0
$$

where

$$
\begin{aligned}
& {\left[\begin{array}{c|c}
T_{1} & T_{2} \\
\hline T_{3} & T_{4}
\end{array}\right] }=\left[\begin{array}{c|c}
\Omega_{i 1} & B_{i} \\
\hline Y_{i} & 0
\end{array}\right], \\
& \Psi_{i 1}-\left(B_{i} \Delta Y_{i}\right)^{\star} \leq 0,
\end{aligned}
$$

where

$$
\begin{aligned}
{\left[\begin{array}{c|c}
T_{1} & T_{2} \\
\hline T_{3} & T_{4}
\end{array}\right] } & =\left[\begin{array}{c|c}
\Psi_{i 1} & -B_{i} \\
\hline Y_{i} & 0
\end{array}\right], \\
\Phi_{i}+\left(B_{i} \Delta Y_{i}\right)^{\star} & <0,
\end{aligned}
$$

where

$$
\begin{aligned}
{\left[\begin{array}{c|c}
T_{1} & T_{2} \\
\hline T_{3} & T_{4}
\end{array}\right] } & =\left[\begin{array}{c|c}
\Phi_{i} & B_{i} \\
\hline Y_{i} & 0
\end{array}\right], \\
\Phi_{i} & =\Phi_{i 1}+\sum_{j \in \mathbb{S}, j \neq i} \frac{\pi_{i j} \gamma_{j}^{2}}{\mu_{j}} X_{i}^{2}
\end{aligned}
$$

Because of $\Delta=\Delta^{T}$ in addition to $\underline{\Delta}=0_{q \times q}$ and $\bar{\Delta}=I_{q}$, it is concluded that $W=0_{q \times q}$ and $S_{i} \in \mathbb{R}^{q \times q}$. Based on (20) and (22), one gets

$$
\left\|\left(A_{i} X_{i}+B_{i} \Delta Y_{i}\right)^{\star}\right\| \leq \frac{2 \ln \gamma_{i}}{d_{i}} \mu_{i} .
$$

From condition (13), we have

$$
X_{i}^{-1} \leq \mu_{i}^{-1} I
$$

implying

$$
\left\|X_{i}^{-1}\right\| \leq \mu_{i}^{-1}
$$

By considering representation (15), (25) is equivalent to

$$
\left\|\left(\bar{A}_{i} X_{i}\right)^{\star}\right\| \mu_{i}^{-1} \leq \frac{2 \ln \gamma_{i}}{d_{i}} .
$$

Then, it is obtained that

$$
\left\|\left(\bar{A}_{i} X_{i}\right)^{\star}\right\|\left\|X_{i}^{-1}\right\| \leq\left\|\left(\bar{A}_{i} X_{i}\right)^{\star}\right\| \mu_{i}^{-1} \leq \frac{2 \ln \gamma_{i}}{d_{i}} .
$$

It is further derived that

$$
\left\|\bar{A}_{i}\right\|=\left\|\bar{A}_{i} X_{i} X_{i}^{-1}\right\| \leq\left\|\bar{A}_{i} X_{i}\right\|\left\|X_{i}^{-1}\right\| \leq \frac{\ln \gamma_{i}}{d_{i}} .
$$

Then, we get

$$
e^{\left\|\bar{A}_{i}\right\| d_{i}} \leq \gamma_{i}
$$

Based on this, condition (19) is guaranteed by

$$
\left(\bar{A}_{i} X_{i}\right)^{\star}+\pi_{i i} X_{i}+\sum_{j \in \mathbb{S}, j \neq i} \frac{\pi_{i j} \gamma_{j}^{2}}{\mu_{j}} X_{i}^{2}<0,
$$

which could be implied by condition (12). This completes the proof.

Remark 5. As for stability problem, [4] firstly presented a necessary and sufficient condition. However, when the system synthesis problems such as stabilization are mentioned, there are few results available. The main reason is that some nonlinear terms will be inevitably encountered, which are not handled easily and directly. From the proof of Theorem 4, such nonlinear terms encountered in condition (7) have been done suitably. Though the large quantity of fault combination, fixed, and random dwell time are included, sufficient existence conditions for fault-tolerant controller (4) are given in terms of LMIs, which are more general than ones in [38]. Moreover, the established conditions are fault-free. In other words, instead of $2^{q}$ combinations involved, only two special cases of no fault and all fault are taken into account, which are fewer than ones in [39-41]. Based on these facts, it is said that the conditions given in this theorem have small computation complexity and could be solved directly and easily.

From Theorem 4, it is seen that the desired controller (4) is mode-dependent and needs its operation mode available online. It is very known that this assumption will be limited in some practical applications. In order to deal with the general condition, a mode-independent controller is usually designed and described as

$$
u(t)=\Delta(t) K x(t),
$$

where $K$ is the common control gain to be determined.

Theorem 6. For given system (1), there exists a modeindependent FTC (33) such that the resulting system is stochastically stable, if for given scalars $\gamma_{i}>1$, and $\mu_{i}>0$, there exist matrices $X_{i}>0, G, Y$, and $S_{i}$ satisfying (13) and

$$
\begin{aligned}
& {\left[\begin{array}{ccc}
\Omega_{i 1} & \Xi_{i} & \widehat{\Omega}_{i 2} \\
* & (-G)^{\star} & Y^{T} \\
* & * & \left(-S_{i}\right)^{\star}
\end{array}\right] \leq 0,} \\
& {\left[\begin{array}{ccc}
\Psi_{i 1} & \Xi_{i} & \widehat{\Psi}_{i 2} \\
* & (-G)^{\star} & Y^{T} \\
* & * & \left(-S_{i}\right)^{\star}
\end{array}\right] \leq 0,}
\end{aligned}
$$

$$
\left[\begin{array}{cccc}
\Phi_{i 1} & \Xi_{i} & \widehat{\Omega}_{i 2} & \Phi_{i 2} \\
* & (-G)^{\star} & Y^{T} & 0 \\
* & * & \left(-S_{i}\right)^{\star} & 0 \\
* & * & * & \Phi_{i 3}
\end{array}\right]<0
$$


where

$$
\begin{gathered}
\widehat{\Omega}_{i 2}=Y^{T}+B_{i} S_{i}^{T}, \\
\widehat{\Psi}_{i 2}=Y^{T}-B_{i} S_{i}^{T}, \\
\Xi_{i}=X_{i}-G^{T} .
\end{gathered}
$$

The other variables are given in Theorem 4. Then, the gain of controller (33) is computed by

$$
K=Y G^{-1} .
$$

Proof. Similar to the proof of Theorem 4, it is concluded that conditions (34)-(36) could imply

$$
\left[\begin{array}{cc}
\Omega_{i 1} & \Xi_{i} \\
* & (-G)^{\star}
\end{array}\right]+\left(\left[\begin{array}{c}
B_{i} \\
0
\end{array}\right] \Delta\left[\begin{array}{ll}
Y & Y
\end{array}\right]\right)^{\star} \leq 0,
$$

where

$$
\begin{aligned}
& {\left[\begin{array}{c|c}
T_{1} & T_{2} \\
\hline T_{3} & T_{4}
\end{array}\right]=\left[\begin{array}{cc|c}
\Omega_{i 1} & \Xi_{i} & B_{i} \\
* & (-G)^{\star} & 0 \\
\hline Y & Y & 0
\end{array}\right],} \\
& {\left[\begin{array}{cc}
\Psi_{i 1} & \Xi_{i} \\
* & (-G)^{\star}
\end{array}\right]+\left(\left[\begin{array}{c}
-B_{i} \\
0
\end{array}\right] \Delta\left[\begin{array}{ll}
Y & Y
\end{array}\right]\right)^{\star} \leq 0,}
\end{aligned}
$$

where

$$
\begin{aligned}
& {\left[\begin{array}{c|c}
T_{1} & T_{2} \\
\hline T_{3} & T_{4}
\end{array}\right]=\left[\begin{array}{cc|c}
\Psi_{i 1} & \Xi_{i} & -B_{i} \\
* & (-G)^{\star} & 0 \\
\hline Y & Y & 0
\end{array}\right],} \\
& {\left[\begin{array}{ccc}
\Phi_{i 1} & \Xi_{i} & \Phi_{i 2} \\
* & (-G)^{\star} & 0 \\
* & * & \Phi_{i 3}
\end{array}\right]+\left(\left[\begin{array}{c}
B_{i} \\
0 \\
0
\end{array}\right] \Delta\left[\begin{array}{lll}
Y & Y & 0
\end{array}\right]\right)^{\star}<0,}
\end{aligned}
$$

where

$$
\left[\begin{array}{l|l}
T_{1} & T_{2} \\
\hline T_{3} & T_{4}
\end{array}\right]=\left[\begin{array}{ccc|c}
\Phi_{i 1} & \Xi_{i} & \Phi_{i 2} & B_{i} \\
* & (-G)^{\star} & 0 & 0 \\
* & * & \Phi_{i 3} & 0 \\
\hline Y & Y & 0 & 0
\end{array}\right] .
$$

Without loss of generality, we only consider (43) in detail. By using Lemma 3 and the Schur complement lemma, it is got that the following condition

$$
\left[\begin{array}{cc}
\Phi_{i}+\left(B_{i} \Delta Y\right)^{\star} & \Xi_{i}+B_{i} \Delta Y \\
* & (-G)^{\star}
\end{array}\right]<0
$$

is guaranteed by condition (43). Moreover, it is concluded from (36) that matrix $G$ is nonsingular. Based on representation (38), it is claimed that

$$
\widehat{\Phi}_{i}+\sum_{j \in \mathbb{S}, j \neq i} \frac{\pi_{i j} \gamma_{j}^{2}}{\mu_{j}} X_{i}^{2}<0,
$$

where

$$
\widehat{\Phi}_{i}=\left(A_{i} X_{i}+B_{i} \Delta Y_{i}\right)^{\star}+\pi_{i i} X_{i}
$$

is implied by pre- and postmultiplying both sides of (45) with

$$
\left[\begin{array}{ll}
I & B_{i} \Delta K
\end{array}\right]
$$

and its transpose, respectively. The next is similar to the proof of (32). On the other hand, as for conditions (39) and (41), it is concluded that they can imply conditions (20) and (22) by pre- and postmultiplying both sides with $\left[\begin{array}{ll}I & B_{i} \Delta K\end{array}\right]$ and $\left[I-B_{i} \Delta K\right]$, respectively. The next steps are similar to the ones in Theorem 4 . This completes the proof.

Remark 7. In order to deal with the mode-independent control problem, a simple and direct way is to select a common Lyapunov function for all modes. Though the gain of controller or filter could be get without any mode information, the choice of mode-independent Lyapunov function usually brings larger conservatism, which may make the design of mode-independent controller fail. In this case, a better way is to make the requirements of mode-independent controller and mode-dependent Lyapunov function be satisfied simultaneously. From Theorem 6, it is seen that such requirements could be satisfied well by letting mode-dependent matrix $X_{i}$ and common matrix $G$. In other words, the conservatism of the obtained results could be reduced, while the goal of modeindependent control could also be achieved.

\section{Numerical Examples}

Example 1. Consider a continuous-time random switching system of form (1) with $\eta(t) \in \mathbb{S}=\{1,2\}$, whose parameters are described as follows:

$$
\begin{aligned}
& A_{1}=\left[\begin{array}{cc}
-1.5 & 0.1 \\
-1 & -2
\end{array}\right], \\
& B_{1}=\left[\begin{array}{cc}
-1 & 0.3 \\
0.4 & 1
\end{array}\right], \\
& A_{2}=\left[\begin{array}{cc}
-2.1 & 0 \\
0.2 & -2.5
\end{array}\right], \\
& B_{2}=\left[\begin{array}{cc}
0.1 & -0.2 \\
0.5 & -2
\end{array}\right] .
\end{aligned}
$$

The transition rate matrix is given as

$$
\Pi=\left[\begin{array}{cc}
-1.2 & 1.2 \\
0.5 & -0.5
\end{array}\right] .
$$

Without loss of generality, the fixed dwell time of such two subsystems is assumed to be $d_{1}=0.1$ and $d_{2}=0.2$, respectively. As for this example, one could design a stabilizing controller by solving a set of LMIs. In this section, we will compare two types of controller: the standard mode-dependent controller (" $S$ " as the subscript, " $i$ " as the superscript) and 
TABLE 1: Stability analysis of system closed by two types of controllers.

\begin{tabular}{llccc}
\hline$\Delta$ & \multicolumn{4}{c}{ Combinations $(1,2,3,4)$} \\
\hline$\delta_{1}$ & 0 & 0 & 1 & 1 \\
$\delta_{2}$ & 0 & 1 & 0 & 1 \\
$K_{S}^{i}$ & $s$ & $u$ & $u$ & $s$ \\
$K_{\mathrm{FTS}}^{i}$ & $s$ & $s$ & $s$ & $s$ \\
\hline
\end{tabular}

the mode-dependent fault-tolerant controller ("FTC" as the subscript, " $i$ " as the superscript). Particularly, the existence conditions of such two controllers are both LMIs, which are described in conditions (20), (22), (23), and (25) with $\Delta=I_{q}$ and conditions in Theorem 4, respectively. By Theorem 4 with $\gamma_{1}=1.6, \gamma_{2}=2, \mu_{1}=0.1$, and $\mu_{2}=0.3$, we have the gains of the mode-dependent fault-tolerant controller (4) computed as

$$
\begin{aligned}
& K_{\mathrm{FTC}}^{1}=\left[\begin{array}{cc}
0.8033 & -0.0584 \\
-0.1135 & 0.1752
\end{array}\right], \\
& K_{\mathrm{FTC}}^{2}=\left[\begin{array}{ll}
-4.1769 & -0.9585 \\
-0.9030 & -0.2501
\end{array}\right] .
\end{aligned}
$$

The gains of standard mode-dependent controller are given as

$$
\begin{aligned}
K_{S}^{1} & =\left[\begin{array}{cc}
-277.9190 & -724.2014 \\
-786.8851 & 247.5663
\end{array}\right], \\
K_{S}^{2} & =\left[\begin{array}{rr}
-4.7291 & -1.1025 \\
-1.1025 & -0.3494
\end{array}\right],
\end{aligned}
$$

which are similar to the ones in [38]. After applying the above controllers, respectively, the stability effects of the resulting closed-loop system are given in Table 1. Here, four types of fault combinations are contained in $\Delta$. In this table, " $s$ " represents the stable closed-loop system, while " $u$ " denotes that the closed-loop system is unstable. Since the above standard mode-dependent controller is without considering faults, the resulting closed-loop system will be unstable for two types of fault combinations. To the contrary, the resulting system closed by the designed mode-dependent fault-tolerant controller (FTC) is always stable. Moreover, though the existence conditions for the desired fault-tolerant controller are within LMI framework, only two special cases of complete fault and no fault are taken into account. Instead of four types of fault combinations involved such as [39-41], the complexity of computation could be reduced; particularly system dimension $n$ or amount of subsystems $N$ is large. Even for a simple case that system dimension $n=2$ and number of subsystems $N=2$, there will be 4 fault combinations, where the amount of LMIs is 8 . In other words, the amount of the fault combinations or system dimension $n$ in addition to quantity of operation mode $N$ has a very large influence on the complexity of the calculation. Under the initial condition $x_{0}=\left[\begin{array}{ll}1 & -1\end{array}\right]^{T}$, the state response of the resulting closed-loop system by the standard mode-dependent controller is given

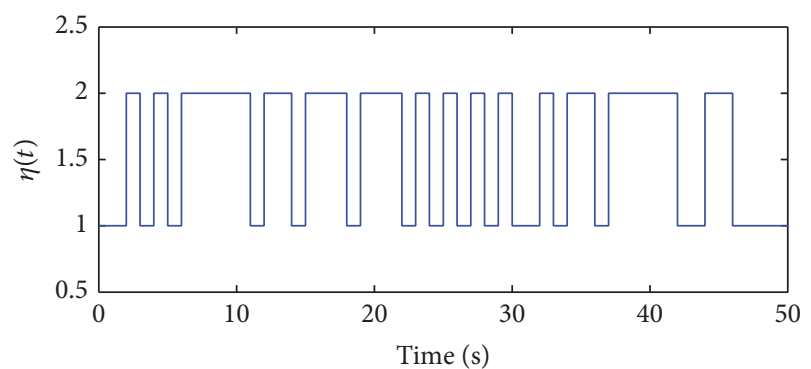

(a)

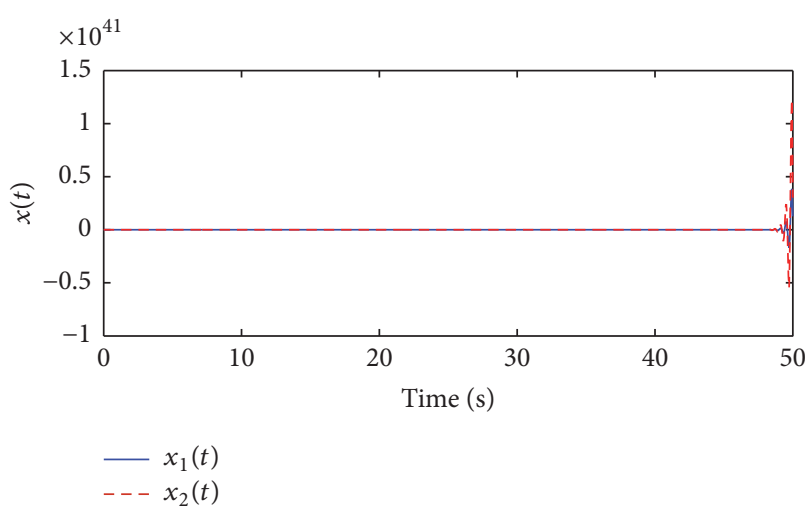

(b)

FIGURE 1: Simulation of system closed by mode-dependent controller.

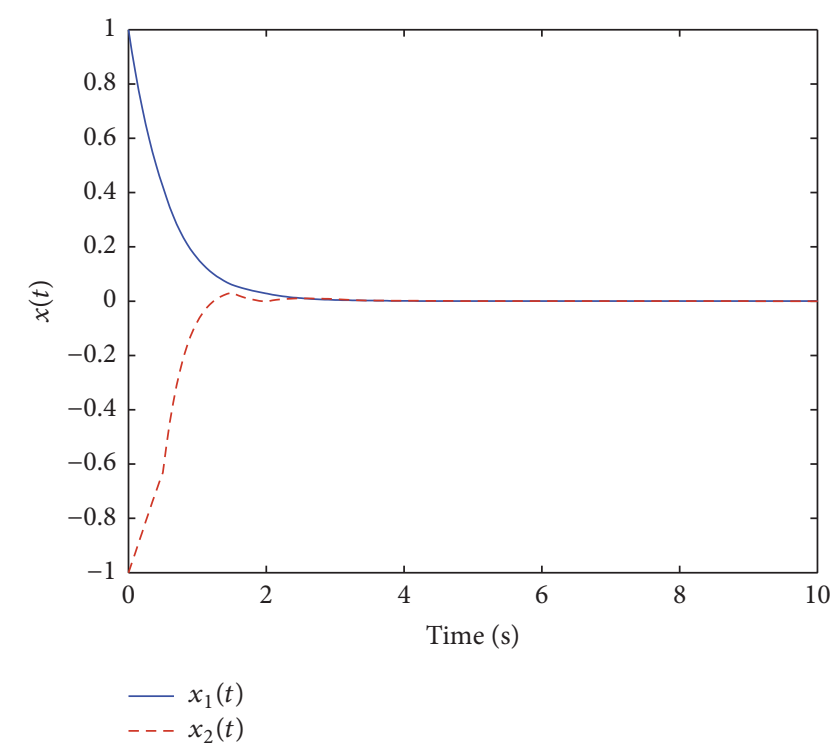

FIGURE 2: State response of system closed by mode-dependent FTC.

in Figure 1(b), while Figure 1(a) is simulation of operation mode. From this simulation, it is said that the fault of controller plays a negative effect to system and could make the system unstable. To the contrary, after applying the above mode-dependent fault-tolerant controller, one could get the state response given in Figure 2. It is obvious that the resulting closed-loop system is stable though there are faults in the desired controller. Moreover, when system mode is 


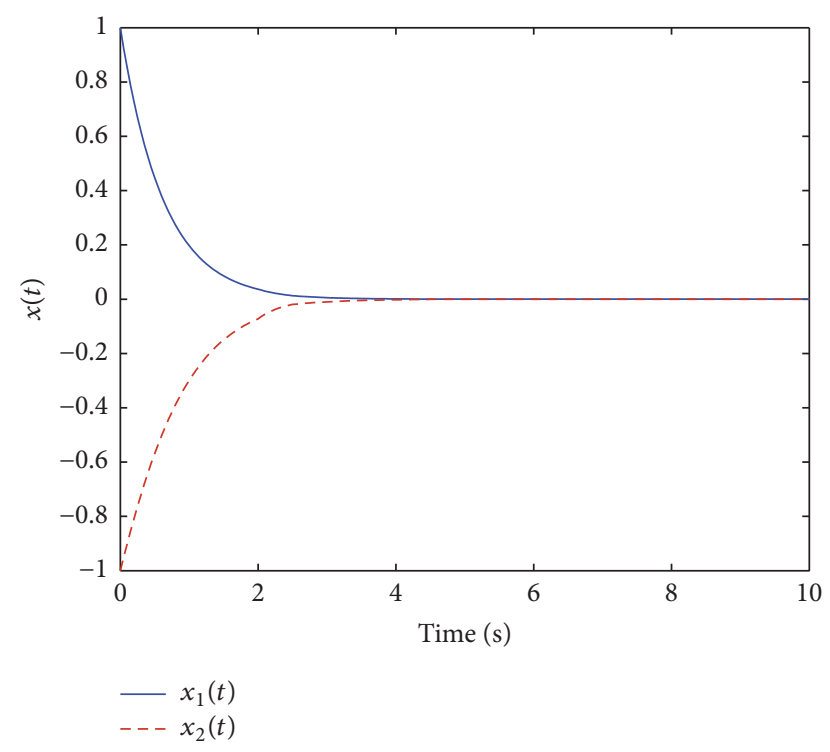

Figure 3: The curves of system closed by mode-independent FTC.

unavailable, a kind of mode-independent fault-tolerant controller (33) could be designed by Theorem 6, and its gain is computed as

$$
K_{\mathrm{FTC}}=\left[\begin{array}{ll}
0.2595 & 0.0459 \\
0.0616 & 0.0422
\end{array}\right] .
$$

The corresponding matrices of Lyapunov function are computed as

$$
\begin{aligned}
& X_{1}=P_{1}^{-1}=\left[\begin{array}{cc}
0.1549 & -0.0125 \\
-0.0125 & 0.1738
\end{array}\right], \\
& X_{2}=P_{2}^{-1}=\left[\begin{array}{cc}
0.3185 & -0.0054 \\
-0.0054 & 0.3450
\end{array}\right] .
\end{aligned}
$$

It is seen that the obtained controller is mode-independent, while the corresponding Lyapunov function is mode-dependent. Because of this, without selecting a common Lyapunov function, the solvable set of mode-independent controller will be larger. Thus, the results will be less conservative than the ones obtained by mode-independent Lyapunov functions. Under the same fault combinations and the initial condition, we could obtain the state response of the resulting closedloop system given in Figure 3 and stable too. Based on these simulations, it is said that our methods based on fault-tolerant controller are superior to ones without considering faults.

\section{Conclusions}

In this paper, the stabilization problem of continuous-time random switching systems has been realized by a fault-tolerant controller, where both fixed and random dwell time are included. Based on the robust method, sufficient conditions for both mode-dependent and mode-independent controllers are established in terms of LMIs, which are also faultfree. Because of all the results without fault information, they are with smaller computation complexity. Compared with the ones obtained by the traditional approaches, the given conditions have fewer LMIs and could be solved easily and directly. Finally, an example has been used to demonstrate the effectiveness and superiority of the proposed methods.

\section{Conflicts of Interest}

The authors declare that there are no conflicts of interest regarding the publication of this paper.

\section{Acknowledgments}

This work was supported by the National Natural Science Foundation of China under Grants 61374043 and 61473140, the Program for Liaoning Excellent Talents in University under Grant LJQ2013040, and the Natural Science Foundation of Liaoning Province under Grant 2014020106.

\section{References}

[1] E.-K. Boukas, Control of Singular Systems with rAndom Abrupt Changes, Communications and Control Engineering Series, Springer, Berlin, Germany, 2008.

[2] S.-L. Kong and Z.-S. Zhang, "Optimal control of stochastic system with Markovian jumping and multiplicative noises," IEEE Transactions on Automatic Control, vol. 38, no. 7, pp. 1113-1118, 2012.

[3] W. Li and Z. Wu, "Output tracking of stochastic high-order nonlinear systems with Markovian switching," IEEE Transactions on Automatic Control, vol. 58, no. 6, pp. 1585-1590, 2013.

[4] J. Xiong, J. Lam, Z. Shu, and X. Mao, "Stability analysis of continuous-time switched systems with a random switching signal," IEEE Transactions on Automatic Control, vol. 59, no. 1, pp. 180186, 2014.

[5] J. Xia, C. Sun, X. Teng, and H. Zhang, "Delay-segment-dependent robust stability for uncertain discrete stochastic Markovian jumping systems with interval time delay," International Journal of Systems Science, vol. 45, no. 3, pp. 271-282, 2014.

[6] R. Rakkiyappan, S. Lakshmanan, R. Sivasamy, and C. P. Lim, "Leakage-delay-dependent stability analysis of Markovian jumping linear systems with time-varying delays and nonlinear perturbations," Applied Mathematical Modelling. Simulation and Computation for Engineering and Environmental Systems, vol. 40, no. 7-8, pp. 5026-5043, 2016.

[7] Y. Ding and H. Liu, "Stability analysis of continuous-time Markovian jump time-delay systems with time-varying transition rates," Journal of the Franklin Institute, vol. 353, no. 11, pp. 24182430, 2016

[8] B. Y. Park, N. K. Kwon, and P. Park, "Stabilization of Markovian jump systems with incomplete knowledge of transition probabilities and input quantization," Journal of the Franklin Institute, vol. 352, no. 10, pp. 4354-4365, 2015.

[9] G. Wang, Q. Zhang, and C. Yang, "Stabilization of singular Markovian jump systems with time-varying switchings," Information Sciences, vol. 297, pp. 254-270, 2015.

[10] G. Wang, "Mode-independent control of singular Markovian jump systems: a stochastic optimization viewpoint," Applied Mathematics and Computation, vol. 286, pp. 155-170, 2016. 
[11] Z. Chen and Q. Huang, "Globally exponential stability and stabilization of interconnected Markovian jump system with mode-dependent delays," International Journal of Systems Science. Principles and Applications of Systems and Integration, vol. 47, no. 1, pp. 14-31, 2016.

[12] S. Xu, J. Lam, and X. Mao, "Delay-dependent $\mathrm{H}_{\infty}$ control and filtering for uncertain Markovian jump systems with timevarying delays," IEEE Transactions on Circuits and Systems I: Regular Papers, vol. 54, no. 9, pp. 2070-2077, 2007.

[13] G. Wang, P. Zhang, and Q. Zhang, "A generalized robust $\mathrm{H}_{\infty}$ filtering for singular Markovian jump systems and its applications," International Journal of Robust and Nonlinear Control, vol. 24, no. 18, pp. 3491-3507, 2014.

[14] C. X. Guan, Z. Y. Fei, Z. C. Li, and Y. L. Xu, "Improved $H_{\infty}$ filter design for discrete-time Markovian jump systems with timevarying delay," Journal of the Franklin Institute, vol. 353, no. 16, pp. 4156-4175, 2016.

[15] L. Wu, X. Su, and P. Shi, "Output feedback control of markovian jump repeated scalar nonlinear systems," IEEE Transactions on Automatic Control, vol. 59, no. 1, pp. 199-204, 2014.

[16] R. Yang, G.-P. Liu, P. Shi, C. Thomas, and M. V. Basin, "Predictive output feedback control for networked control systems," IEEE Transactions on Industrial Electronics, vol. 61, no. 1, pp. 512-520, 2014.

[17] Y. Wei, J. Qiu, and S. Fu, "Mode-dependent nonrational output feedback control for continuous-time semi-Markovian jump systems with time-varying delay," Nonlinear Analysis: Hybrid Systems, vol. 16, pp. 52-71, 2015.

[18] H. Fan, B. Liu, W. Wang, and C. Wen, "Adaptive fault-tolerant stabilization for nonlinear systems with Markovian jumping actuator failures and stochastic noises," Automatica, vol. 51, pp. 200-209, 2015.

[19] M. Liu, D. W. Ho, and P. Shi, "Adaptive fault-tolerant compensation control for Markovian jump systems with mismatched external disturbance," Automatica, vol. 58, pp. 5-14, 2015.

[20] H. Li, P. Shi, D. Yao, and L. Wu, "Observer-based adaptive sliding mode control for nonlinear Markovian jump systems," Automatica, vol. 64, pp. 133-142, 2016.

[21] R. Rakkiyappan, A. Chandrasekar, J. H. Park, and O. M. Kwon, "Exponential synchronization criteria for Markovian jumping neural networks with time-varying delays and sampled-data control," Nonlinear Analysis. Hybrid Systems, vol. 14, pp. 16-37, 2014.

[22] H. Shen, J. H. Park, Z.-G. Wu, and Z. Zhang, "Finite-time Ho synchronization for complex networks with semi-Markov jump topology," Communications in Nonlinear Science and Numerical Simulation, vol. 24, no. 1-3, pp. 40-51, 2015.

[23] G. Wang and Q. Zhang, "Robust control of uncertain singular stochastic systems with Markovian switching via proportionalderivative state feedback," IET Control Theory \& Applications, vol. 6, no. 8, pp. 1089-1096, 2012.

[24] P. Shi, Y. Yin, F. Liu, and J. Zhang, "Robust control on saturated Markov jump systems with missing information," Information Sciences, vol. 265, pp. 123-138, 2014.

[25] H. Yang, H. Li, F. Sun, and Y. Yuan, "Robust control for Markovian jump delta operator systems with actuator saturation," European Journal of Control, vol. 20, no. 4, pp. 207-215, 2014.

[26] Q. Zhou, D. Yao, J. Wang, and C. Wu, "Robust control of uncertain semi-Markovian jump systems using sliding mode control method," Applied Mathematics and Computation, vol. 286, pp. 72-87, 2016.
[27] X. Zhang, T. Parisini, and M. M. Polycarpou, "Adaptive fault-tolerant control of nonlinear uncertain systems: an informationbased diagnostic approach," IEEE Transactions on Automatic Control, vol. 49, no. 8, pp. 1259-1274, 2004.

[28] H. Alwi and C. Edwards, "Fault tolerant control using sliding modes with on-line control allocation," Automatica, vol. 44, no. 7, pp. 1859-1866, 2008.

[29] Q. Yang, S. S. Ge, and Y. Sun, "Adaptive actuator fault tolerant control for uncertain nonlinear systems with multiple actuators," Automatica, vol. 60, pp. 92-99, 2015.

[30] H. Niemann and J. Stoustrup, "Passive fault tolerant control of a double inverted pendulum - a case study," Control Engineering Practice, vol. 13, no. 8, pp. 1047-1059, 2005.

[31] M. Benosman and K. Y. Lum, "Passive actuators' fault tolerant control for affine nonlinear systems," IEEE Transactions on Control Systems Technology, vol. 18, no. 1, pp. 152-163, 2010.

[32] F. Shi and R. J. Patton, "Fault estimation and active fault tolerant control for linear parameter varying descriptor systems," International Journal of Robust and Nonlinear Control, vol. 25, no. 5, pp. 689-706, 2015.

[33] F. R. Segundo Sevilla, I. M. Jaimoukha, B. Chaudhuri, and P. Korba, "A semidefinite relaxation procedure for fault-tolerant observer design," IEEE Transactions on Automatic Control, vol. 60, no. 12, pp. 3332-3337, 2015.

[34] M. Liu, X. B. Cao, and P. S. Shi, "Fault estimation and tolerant control for fuzzy stochastic systems," IEEE Transactions on Fuzzy Systems, vol. 21, no. 2, pp. 221-229, 2013.

[35] H. Li, H. Gao, P. Shi, and X. Zhao, "Fault-tolerant control of Markovian jump stochastic systems via the augmented sliding mode observer approach," Automatica, vol. 50, no. 7, pp. 18251834, 2014.

[36] L. Chen, X. Huang, and S. Fu, "Observer-based sensor faulttolerant control for semi-Markovian jump systems," Nonlinear Analysis. Hybrid Systems, vol. 22, pp. 161-177, 2016.

[37] D. P. De Farias, J. C. Geromel, J. B. R. Do Val, and O. L. V. Costa, "Output feedback control of Markov jump linear systems in continuous-time," IEEE Transactions on Automatic Control, vol. 45, no. 5, pp. 944-949, 2000.

[38] G. Wang, Q. Zhang, C. Yang, and C. Su, "Stabilization of continuous-time randomly switched systems via the LMI approach," Applied Mathematics and Computation, vol. 266, pp. 527-538, 2015.

[39] M. Staroswiecki, G. Hoblos, and A. Aitouche, "Sensor network design for fault tolerant estimation," International Journal of Adaptive Control and Signal Processing, vol. 18, no. 1, pp. 55-72, 2004.

[40] M. Cattafi, M. Gavanelli, M. Nonato, S. Alvisi, and M. Franchini, "Optimal placement of valves in a water distribution network with CLP(FD)," Theory and Practice of Logic Programming, vol. 11, no. 4-5, pp. 731-747, 2011.

[41] S. Alvisi, M. Franchini, M. Gavanelli, and M. Nonato, "Nearoptimal scheduling of device activation in water distribution systems to reduce the impact of a contamination event," Journal of Hydroinformatics, vol. 14, no. 2, pp. 345-365, 2012. 


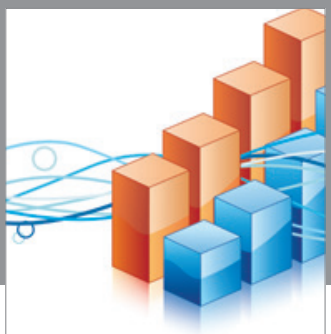

Advances in

Operations Research

vatem alat4

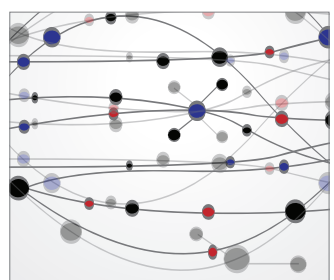

\section{The Scientific} World Journal
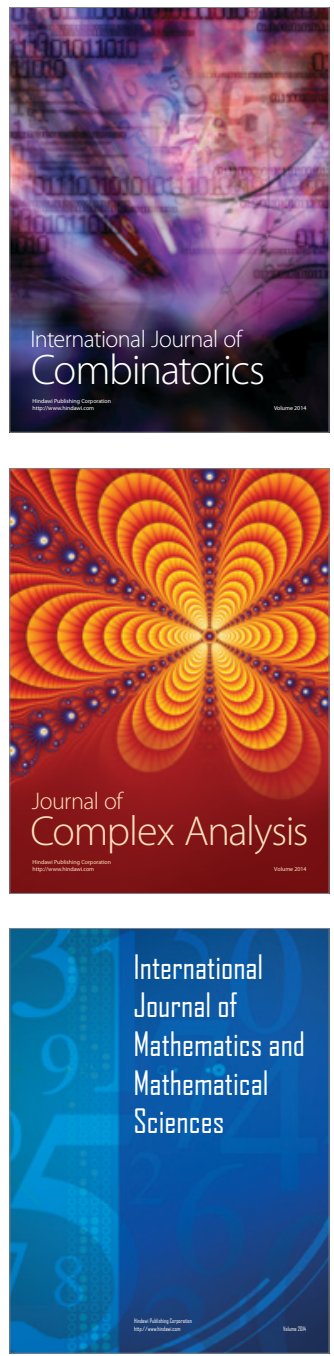
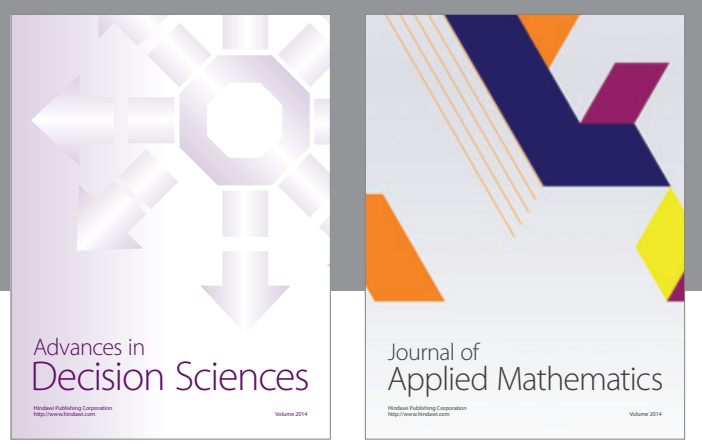

Algebra

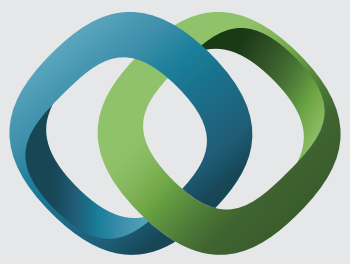

\section{Hindawi}

Submit your manuscripts at

https://www.hindawi.com
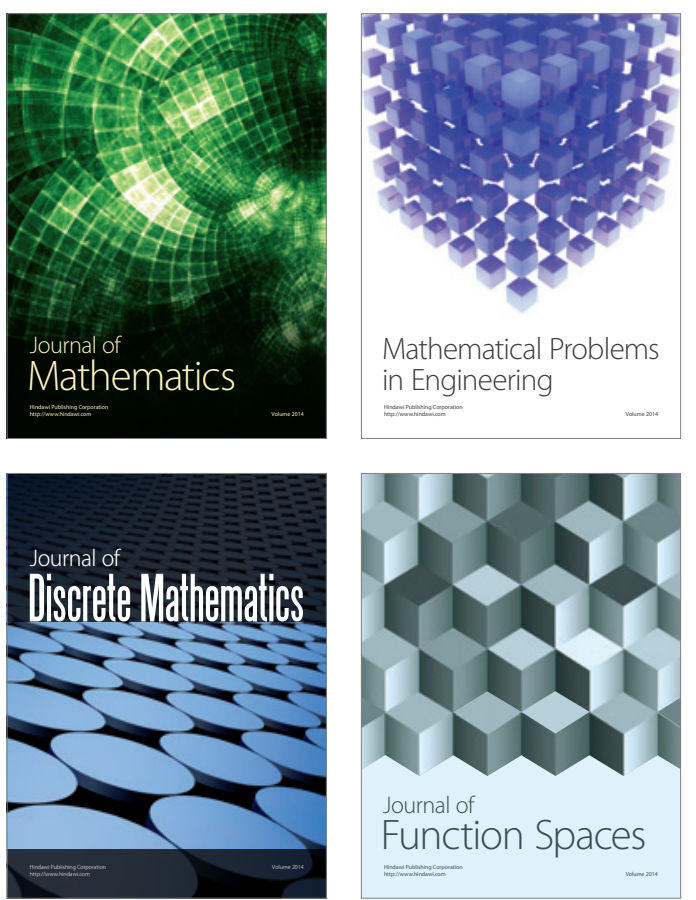

Mathematical Problems in Engineering
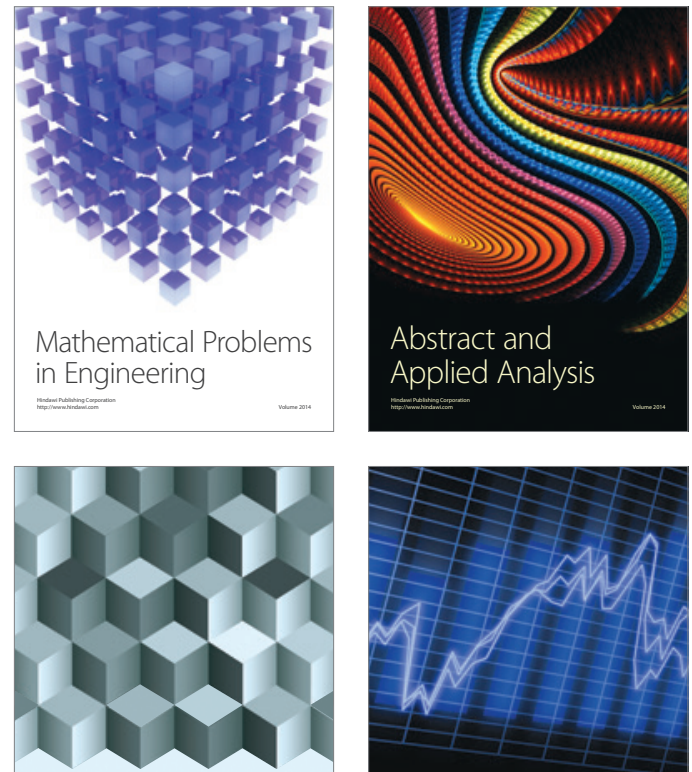

Journal of

Function Spaces

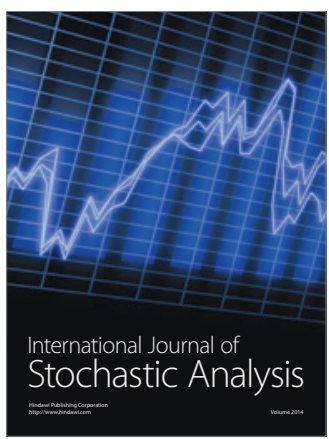

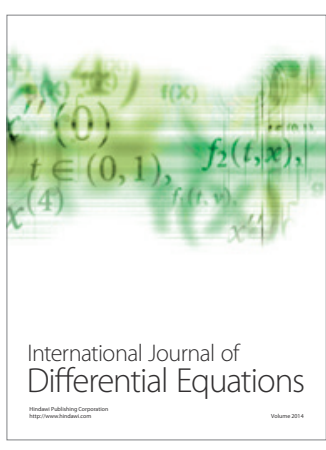
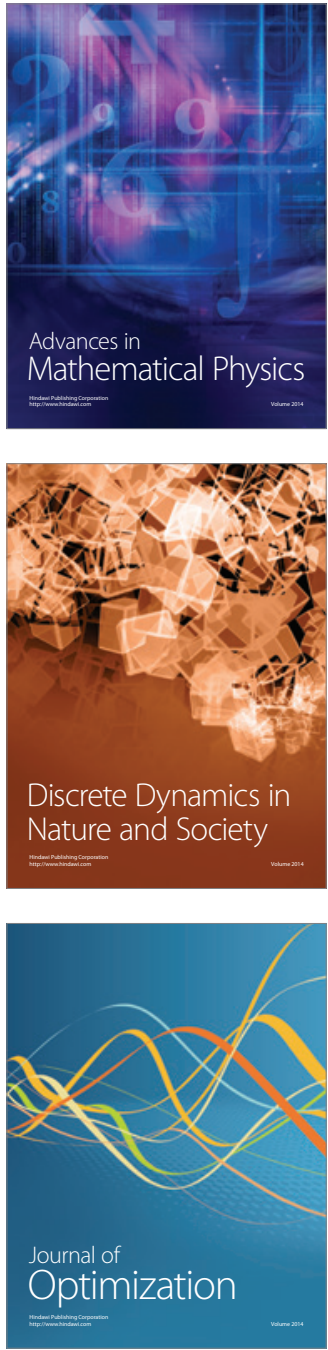